\title{
ST Algorithm for Medical Diagnostic Reasoning
}

\author{
Irosh Fernando and Frans A. Henskens
}

\begin{abstract}
The authors have previously described an approach for medical diagnostic reasoning based on the ST (Select and Test) model introduced by Ramoni and Stefanelli et al. This paper extends the previous approach by introducing the required algorithm for medical expert system development. The algorithm involves a bottom-up and recursive process using logical inferences, abduction, deduction, and induction. Pseudocode for the algorithm, and the data structures involved, are described, and the algorithm's implementation using a small sample knowledgebase and programmed in Java is included in appendixes. Implementation of a successful expert system is a challenging process; development of the necessary algorithm for its inference engine, and definition of a knowledgebase structure that models expert diagnostic reasoning and knowledge, only fulfils the initial step. Challenges associated with the remaining steps of the development process can be identified and dealt with using the CLAP software process model.
\end{abstract}

Index Terms-Medical diagnostic reasoning, medical expert systems, ST model.

\section{INTRODUCTION}

$\mathrm{R}$ EALISATION of medical expert systems has been one of the earliest goals of the AI community. Unfortunately, attempts by major projects such as INTERNIST-I and CADUCEUS have not been successful [1]

One of the reasons for this failure can be understood in relation to the lack of models that capture the depth and complexity of expert medical diagnostic reasoning. Models previously proposed for medical diagnostic reasoning include: scheme-inductive reasoning [2]; hypothetico-deductive reasoning [3]; backward and forward reasoning [4]; pattern recognition [5]; Parsimonious Covering Theory [6]; Information Processing Approach [7]; Process Model for diagnostic reasoning [8]; Certainty Factor model [9]; models based on Bayes Theorem [10-12]; and models based on Fuzzy logic [13-15]. The authors have previously described the limitations of some of these approaches, and proposed an approximate reasoning model for medical diagnostic reasoning [16]. This previously proposed model was based on

Manuscript received August 6, 2013; accepted for publication on September 30, 2013

D. A. I. P. Fernando is with the School of Electrical Engineering and Computer Science, University of Newcastle, NSW 2308, Australia (phone: +61 423281 664; e-mail: irosh.fernando@uon.edu.au).

F. A. Henskens is with the School of Electrical Engineering and Computer Science, University of Newcastle, NSW 2308, Australia (e-mail: frans.henskens@newcastle.edu.au). the epistemological framework (also known as Select and Test (ST) model) for medical diagnostic reasoning proposed by Ramoni and Stefanelli et al. [17].

This paper complements the authors' previous approach by introducing the required algorithm for diagnostic inference. The previously proposed reasoning model requires of at least three layers of knowledgebase entities, namely diagnoses, symptoms and symptom attributes, together with mathematical functions to quantify those entities. In order to improve readability, the algorithm described in this paper has been deliberately simplified by restricting its application to the first two layers only. Extension of the algorithm to incorporate the full model is explained in the Discussion section of this paper.

The remainder of the paper begins with an introduction to the ST Model followed by formalisation of the knowledgebase as a graph consisting of symptoms and diagnoses. Then, the algorithm's pseudocode and the data structures, and its implementation, are described using a sample knowledgebase. Before the paper is concluded, other challenges that are faced in developing successful medical expert systems are briefly outlined, and the CLAP software process model [18] is described as a framework for addressing these challenges in a systematic manner.

\section{Select And Test (ST) Model}

The ST Model describes a cyclical process (Fig. 1), which uses the logical inferences, abduction, deduction, and induction that were described by Charles Peirce [19]. Usually, diagnostic reasoning in clinical contexts begins when a patient reports a symptom or symptoms to their clinician. Whilst these symptoms are well-defined entities in the clinician's mind, patients may use various descriptive terms to describe their symptoms. For example, a patient may use the descriptive term 'a dark cloud over me' to describe the symptom 'low mood'.

The process of mapping these descriptive terms understood by patients onto well-defined symptom entities used in the knowledgebase is known as abstraction. The next step, known as abduction, involves determining all likely diagnoses related to the reported symptoms. Then, for each likely diagnosis, it is necessary to determine if the patient is experiencing other expected symptoms. This is known as deduction. These three steps repeat cyclically until all the required symptoms and diagnoses have been explored. Once this cycle is ended, the final step, induction, occurs. 


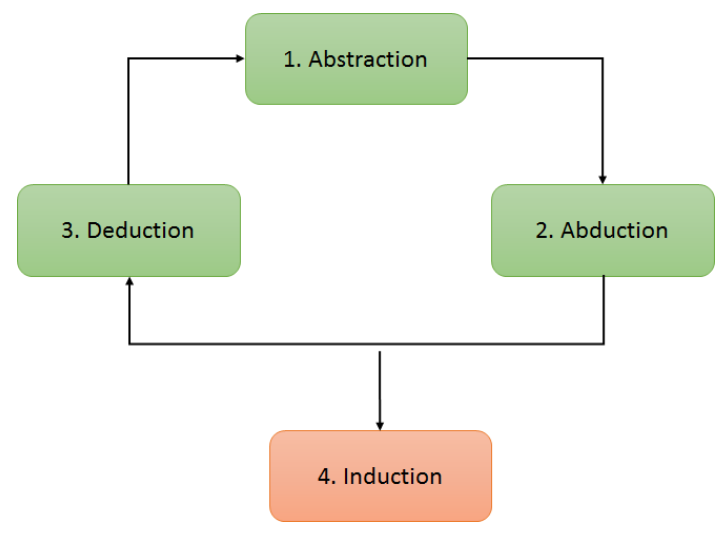

Fig. 1. The ST Model.

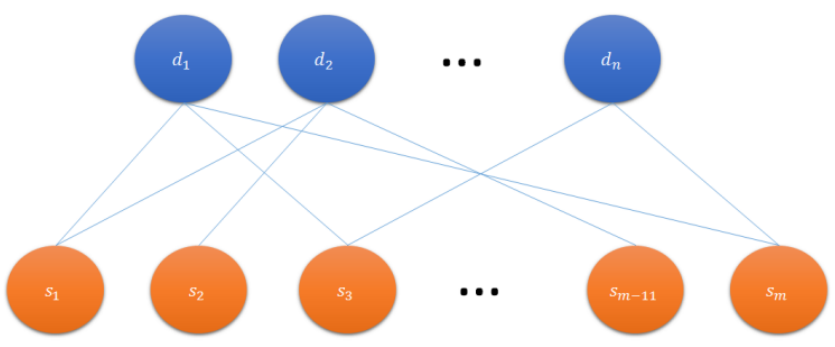

Fig. 2. Simplified knowledgebase representing diagnoses and symptoms only.

Induction involves matching the elicited symptoms with the expected symptoms for each likely diagnosis, thus determining whether the patient is suffering from any of the likely diagnoses. More details of the ST model can be found in the paper published by Ramoni and Stefanelli et al [17].

\section{Formal MODEL For KNOWLEDGEBASE}

By way of formalising the process described above, let us represent all the diagnoses and symptoms in our knowledgebase as sets $D=\left\{d_{1}, d_{2}, \ldots, d_{n}\right\}$ and $S=\left\{s_{1}, s_{2}, \ldots s_{m}\right\}$ respectively. The relationship representing 'given a symptom $s_{i}$ how likely is diagnosis $d_{j}^{\prime}$ is represented as a two-layer graph (Fig. 2), in which each arc is associated with a value $\theta_{i j}$ representing the likelihood $(L)$ that $s_{i}$ implies $d_{j}$; note $0 \leq \theta_{i j} \leq 1$. This can also be represented using the notation $L\left(d_{j}\right.$ $\left.\mid s_{i}\right)=\theta_{i j}$. By way of example, in Fig. 2 the arc connecting $d_{1}$ and $s_{3}$ would have associated likelihood $\theta_{31}$. The knowledgebase consisting of the two layers, symptoms and diagnoses, can be represented as a matrix $\left[\theta_{i j}\right]$.

\section{Select And Test Algorithm}

Medical diagnostic reasoning involves two main steps:

1. search for symptoms,

2. arrive at diagnoses based on the symptoms found in the previous step.
Because of the vastness of the knowledgebase, one of the most challenging aspects of diagnostic reasoning is the symptom search process. It is therefore not uncommon that even an experienced clinician can at times miss a diagnosis because of failure to elicit a key symptom that would have provided an important clue to a diagnosis. If all the symptoms are known, arriving at a diagnosis is relatively easy computationally, depending on the commonly agreed or established diagnostic criteria used in different medical specialities. For example, in psychiatry, if all the symptoms are known, the second step involves matching the elicited symptoms with the diagnostic criteria described in a standard diagnostic manual such as DSM-V [20]. In the ST algorithm, abstraction, abduction and deduction are involved in the first step, and induction is involved in the second step.

The proposed algorithm uses five dynamic data structures, namely symptomsFound, symptomsToBeElicited, symptomsAlreadyElicited, diagnosesToBeElicited and diagnosesAlreadyElicited, which are implemented as linked lists. Also, in order to describe how the algorithm works, a static data structure patientProfile, which an artificial entity that encapsulates all the symptoms actually present in a patient, is used. The nature of the real world diagnostic problem is that the symptoms a patient actually has are initially unknown to the clinician. Symptom searching (the first step) in real world diagnostic reasoning can be conceptualised as an endeavour to find all the content, or at least all the clinically important symptoms, stored in patientProfile. In real world situations patientProfile is a virtual entity because it represents the patient's actual symptoms, which need to be discovered by the clinician when the patient is interviewed.

Details of the abstraction step have also been simplified in this paper. Whilst, in the real world setting, abstraction involves mapping the patient's symptom descriptions to defined knowledgebase entities, this largely mechanical matching process is omitted. Rather, it is assumed that the symptom descriptions stored in patientProfile correspond to the symptom descriptions used in the knowledgebase.

Implementation of abstraction in a real world application would require, for example, the patient informing symptoms by answering closed-ended questions using check boxes in a very basic human computer interface, or via an actual dialog between patient and expert system using natural language capabilities.

The data structures used in a computer-based implementation of the algorithm are described in Fig. 3, and the algorithm itself is shown in Fig. 6.

The ST Algorithm starts when a patient reports a set of initial symptoms that are stored in symptomsFound and diagnosesAlreadyElicited.

Abduction then begins, returning all the diagnoses connected to each symptom stored in symptomsFound. A threshold value likelihoodThreshold in relation to the connection strength between any symptom and related diagnosis can be used to determine which diagnoses are to be retrieved. 


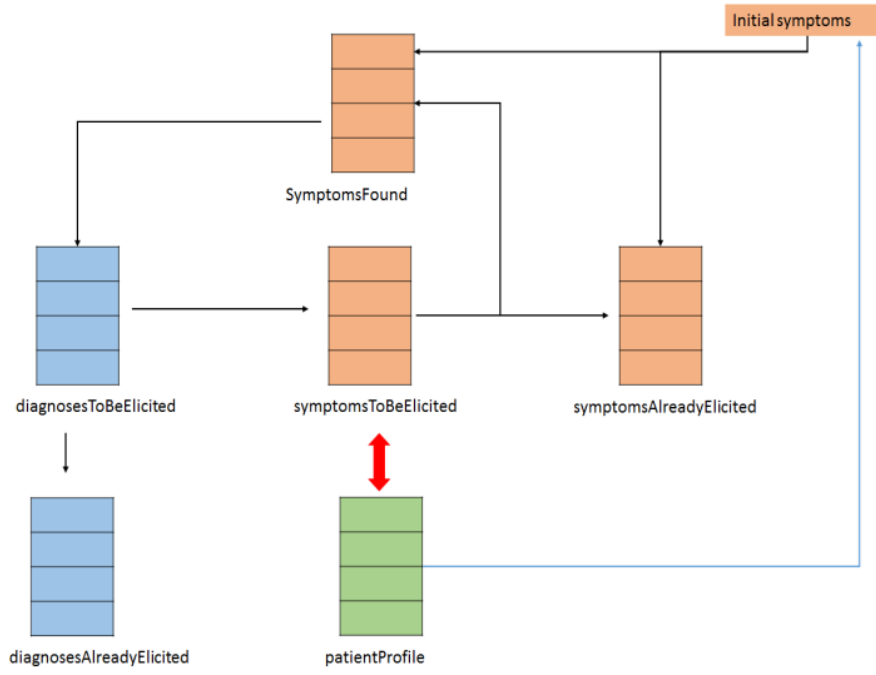

Fig. 3. Data structures used in the ST algorithm.

Accordingly, for any given symptom $s_{i}$ the system will retrieve all the diagnoses $d_{j}$ for which the likelihood that the symptom is caused by the diagnosis is in accordance with $\theta_{i j}>$ likelihoodThreshold.

These diagnoses are stored in the linked list diagnosesToBeElicited; before storing each diagnosis the system checks if it has already been stored in diagnosesAlreadyElicited because of association with a previous symptom, thus avoiding the possibility of duplicate diagnoses.

Next, deduction begins by returning all the expected symptoms connected with each diagnosis stored in diagnosesToBeElicited, after which the diagnosis is removed from diagnosesToBeElicited and transferred into diagnosesAlreadyElicited. All the expected symptoms that are returned for each diagnosis are transferred into diagnosesToBeElicited unless they are already stored in diagnosesAlreadyElicited.

Abstraction then commences, eliciting each symptom stored in symptomsToBeElicited by searching patientProfile. Then the elicited symptom is removed from symptomsToBeElicited and transferred into symptomsAlreadyElicited. If the elicited symptom is found in patientProfile it is stored in symptomsFound.

Finally, induction involves matching diagnostic criteria (i.e. symptoms expected for each diagnosis in diagnosesAlreadyElicited) with the symptoms stored in symptomsFound. If the diagnostic criteria are met, depending on the expected symptoms and the symptoms in symptomsFound then the respective diagnosis is accepted. Otherwise the respective diagnosis is excluded.

\section{AN EXAMPLE AND ITS IMPLEMENTATION}

In order to elaborate the proposed algorithm, let us consider a small knowledge base consisting of twelve symptoms and six diagnoses as described in Fig. 4. The relationships between these diagnoses and symptoms (i.e. $\theta_{i j}$ as described previously) are described in the matrix shown in Fig. 5. Java implementations of this knowledgebase and the algorithm are presented in Appendices 1 and 2, respectively.

\begin{tabular}{|c|l|}
\hline$s_{1}$ & Depressed mood \\
\hline$s_{2}$ & Loss of motivation \\
\hline$s_{3}$ & Weight loss \\
\hline$s_{4}$ & Fatigue \\
\hline$s_{5}$ & Chest discomfort \\
\hline$s_{6}$ & Worrying thoughts \\
\hline$s_{7}$ & Low self-esteem \\
\hline$s_{8}$ & Headache \\
\hline$s_{9}$ & Loss of appetite \\
\hline$s_{10}$ & Hand tremors \\
\hline$s_{11}$ & Hypertension \\
\hline$s_{12}$ & Gastrointestinal bleeding \\
\hline
\end{tabular}

\begin{tabular}{|l|l|l|}
\hline$d_{1}$ & Major Depression & 0.5 \\
\hline$d_{2}$ & Generalized Anxiety Disorder & 0.2 \\
\hline$d_{3}$ & Hyperthyroidism & 0.6 \\
\hline$d_{4}$ & Pheochromocytoma & 0.7 \\
\hline$d_{5}$ & Anaemia & 0.7 \\
\hline$d_{6}$ & Ischaemic Heart Disease & 0.9 \\
\hline
\end{tabular}

Fig. 4. Symptoms and diagnoses to be included in sample knowledgebase.

Details of the induction step are omitted in the implementation; the implementation of this step depends on the diagnostic criteria that are used to match the elicited symptoms with the expected symptoms of diagnoses. In its simplest form, the induction step can be implemented by one to one matching of the expected symptoms with the elicited symptoms. Nonetheless, depending on the diagnosis, it may not be necessary to have all the expected symptoms to make that diagnosis. In such situations, logical expression constructed using $A N D$ and $O R$ operators can be used to formulate diagnostic rules by connecting different combination of symptoms. These diagnostic rules can be enhanced by allowing quantification of the severity of the symptoms elicited in the patient, as described elsewhere [16].

\begin{tabular}{|l|l|l|l|l|l|l|}
\hline & \multicolumn{1}{|c|}{$d_{1}$} & \multicolumn{1}{|c|}{$d_{2}$} & \multicolumn{1}{|c|}{$d_{3}$} & \multicolumn{1}{c|}{$d_{4}$} & \multicolumn{1}{c|}{$d_{5}$} & \multicolumn{1}{c|}{$d_{6}$} \\
\hline$s_{1}$ & 0.9 & 0 & 0.3 & 0 & 0.3 & 0.3 \\
\hline$s_{2}$ & 0.9 & 0 & 0 & 0 & 0.3 & 0 \\
\hline$s_{3}$ & 0.6 & 0 & 0.7 & 0 & 0 & 0 \\
\hline$s_{4}$ & 0.6 & 0.7 & 0.6 & 0 & 0.8 & 0.3 \\
\hline$s_{5}$ & 0 & 0.6 & 0 & 0 & 0 & 0.8 \\
\hline$s_{6}$ & 0 & 0.9 & 0 & 0 & 0 & 0 \\
\hline$s_{7}$ & 0.6 & 0.4 & 0 & 0 & 0 & 0 \\
\hline$s_{8}$ & 0 & 0.6 & 0 & 0.5 & 0 & 0 \\
\hline$s_{9}$ & 0.7 & 0 & 0 & 0 & 0 & 0 \\
\hline$s_{10}$ & 0 & 0.6 & 0.8 & 0 & 0 & 0 \\
\hline$s_{11}$ & 0 & 0 & 0 & 0.9 & 0 & 0.4 \\
\hline$s_{12}$ & 0 & 0.4 & 0.3 & 0 & 0.6 & 0.6 \\
\hline
\end{tabular}

Fig. 5. Representation of the knowledgebase as a matrix, [ $\left.\theta_{i j}\right]$. 


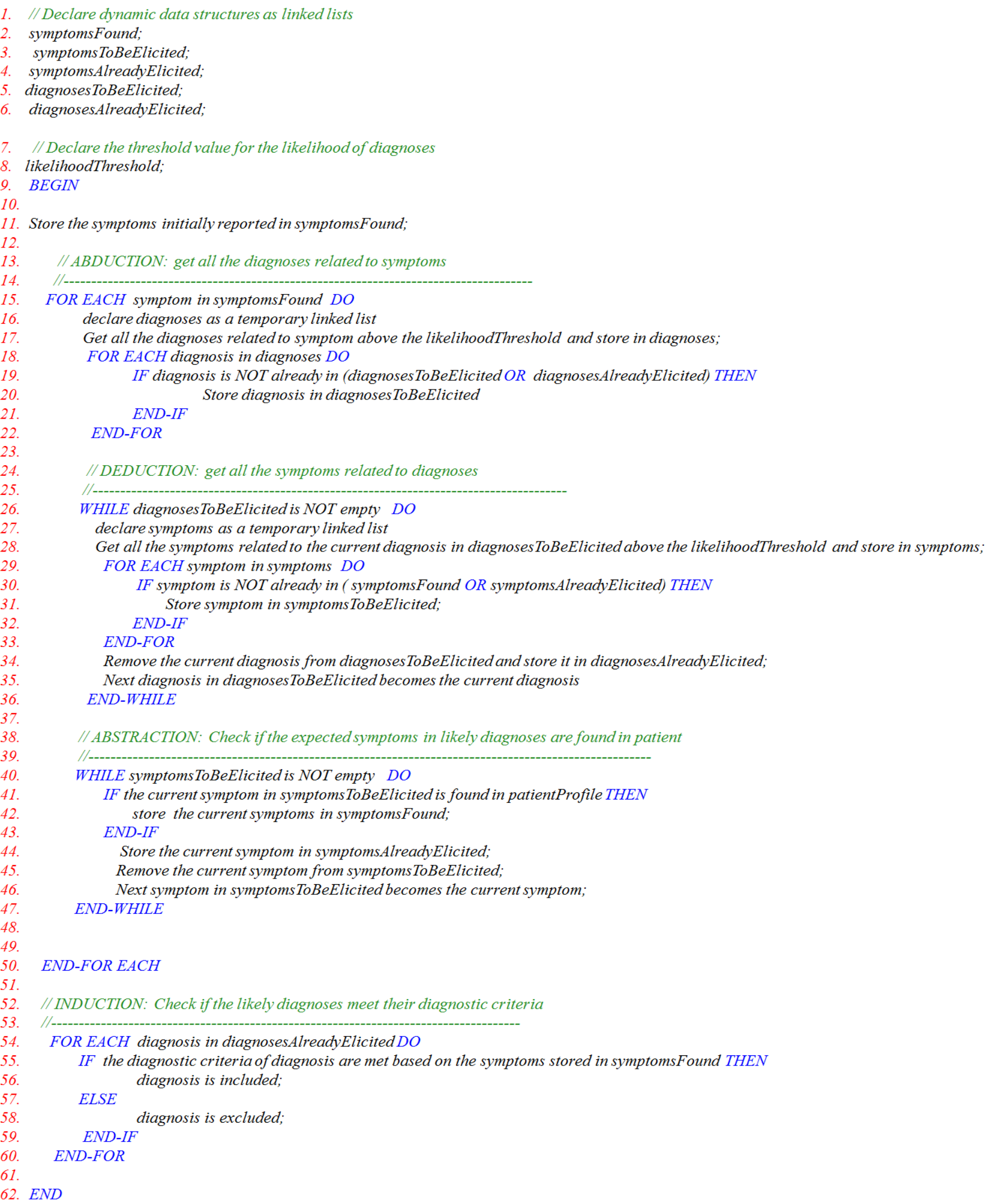

Fig. 6. ST algorithm. 
For example, consider the diagnosis $d_{1}=$ Major Depression, $s_{1}=$ Depressed Mood, and the related symptoms $s_{1}=$ Depressed Mood,$s_{2}=$ Loss of Motivation, $s_{3}=$ Weight Loss, and $s_{7}=$ Low Self Esteem.

Suppose we have a patient who presents with the above symptoms, each with a different level of severity. Let us assume that the severity of these symptoms (i.e. quantification) corresponds to $q_{1}, q_{2}, q_{3}$ and $q_{7}$ respectively. Using threshold values $t_{11}, t_{12}, t_{13}$ and $t_{17}$ respectively for each of these symptoms in relation to $d_{1}$, an example of a diagnostic rule is as follows:

$$
\begin{array}{r}
\text { IF }\left(q_{1}>t_{11} \text { AND } q_{2}>t_{12} \text { AND } q_{3}>t_{13} \text { AND } q_{7}>t_{17}\right) \text { THEN } \\
\text { accepted }\left(d_{1}\right)=\text { TRUE }
\end{array}
$$

where accepted $\left(d_{1}\right)$ indicates whether the diagnostic criteria for $d_{1}$ is met, resulting in its acceptance (or rejection) as a diagnosis. It may require several such diagnostic rules for each diagnosis, and some of the rules may also require the logical operator OR in addition to AND. Developers may have to consult standard diagnostic manuals (for example, DSM V [20] in psychiatry) when formulating the diagnostic rules.

\section{DISCUSSION}

The knowledgebase model and algorithm presented above represent a simplified version of what it is required for effective diagnostic inference in real world settings. Nonetheless, they encapsulate the essential basic characteristics of the reasoning process. This basic structure can be extended and customised according to the characteristics of clinical knowledge in various medical subspecialties (i.e. subdomains). For example, in psychiatry, the knowledgebase may require addition of an extra layer known as clinical phenomenon between the symptoms and diagnoses layers [21]. Also, an extra layer of symptom attributes can be added below the symptoms layer, and each symptom can be quantified using the values associated with the related symptom's attributes using mathematical functions that approximate their relationships, as described elsewhere [16].

In addition to searching for diagnoses related to a given symptom based on likelihood, as described in the algorithm, diagnostic reasoning in the real world setting also involves searching for more critical (i.e. associated with relatively worse consequences if undetected) diagnoses even though their likelihoods seem low based on the patient's reported symptoms. The ST algorithm does an exhaustive search, and therefore can be useful in ruling out more critical diagnoses that can present with rather atypical symptoms. It is possible to enhance ST by introducing a critical value $\delta_{j}$ associated with each diagnosis $d_{j}$ that determines the level of criticality of the diagnosis. Similarly to the likelihoodThreshold described previously, a threshold value criticality Threshold can be used to select diagnoses for which $\delta_{j}>$ criticality Threshold.
The next significant challenge to implementing the algorithm in a practically useful expert system is developing and maintaining a sufficiently large knowledgebase. Because of the vastness of the knowledgebase and the amount of manpower and commitment required to develop and maintain it, a sufficient database has been very difficult to achieve using traditional development methods [22]. For example, despite expending nearly 25-30 person years of work, it has still not been able to complete the knowledgebase of INTERNIST-I, an expert diagnostic system in Internal Medicine [1].

Even if the required knowledgebase were implemented, there are yet more challenges. An important challenge is engaging clinicians, who may often feel threatened by medical expert systems on the grounds they may be intended to duplicate and replace some of their skills [23]. The authors have previously discussed these challenges, and introduced a software process model known as a Collaborative and Layered Approach (CLAP) as a strategy to deal with these challenging issues [18].

The main layers and the activities within each layer of CLAP are shown in Fig. 7, and the reader is encouraged to refer to the main paper on this model for more details [18]. The form of ST algorithm introduced in this paper can be considered as the main product of the conceptual layer, which primarily deals with conceptualising the expert medical reasoning process and the knowledgebase, and then translating into a formal model. The societal layer then deals with engaging clinicians in a collaborative development process, and defining the role of the under-development expert system within the complex modern day organisational structure of healthcare services in which it will be used. Finally, the computational layer deals with software and hardware implementation of the expert system. As an important strategy to overcome the difficulty of developing and maintaining the knowledgebase, the CLAP model suggests use of an online collaborative approach [24], which can be realised due to advancement of Internet-based social networking platforms.

\section{CONCLUSION}

Whilst acknowledging the challenges in developing successful medical expert systems, this paper introduced a simplified version of the algorithm and data structures required for implementing an inference engine and knowledgebase, based on a previously introduced diagnostic reasoning model [16]. Even though there are many diagnostic reasoning models that have been previously introduced, the authors claim that the reasoning model on which the algorithm introduced in ST this paper is designed, is more comprehensive in relation to the overall expert diagnostic reasoning process.

Furthermore, the algorithm closely models the recursive steps that are involved in real world diagnostic reasoning, using logical inferences. Because of the complexity and the space required to describe the full algorithm and its implementation, it was necessary in this paper to simplify the 
algorithm and knowledgebase described. However, the paper still provides the core structure on which, the full model can be built. As a means to identifying and resolving other challenges associated with the development process, the authors suggest use of the CLAP software process model for developing medical expert systems [18].

\section{APPENDICES}

\section{Appendix 1. Java representation of the knowledgebase}

package diagnosticalgorithm; import java.util.ArrayList;

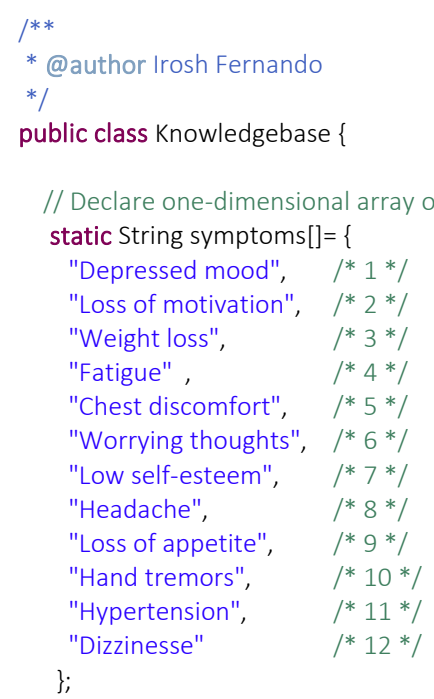

// Declare the knowledgebase as a two dimensional array

static double diag_symp[][] $=\{$

/* symptoms index: $1,2,3,4,5,6,7,8,9,10,11,12$ */ $/ *$ diagnosis index: $1 * /\{0.9,0.9,0.6,0.6,0.0,0.0,0.6,0.0,0.7,0.0,0.0,0.0\}$,

$/ *$ diagnosis index: $2 * /\{0.0,0.0,0.0,0.7,0.6,0.9,0.4,0.6,0.0,0.6,0.0,0.4\}$,

$/ *$ diagnosis index: $3 * /\{0.3,0.0,0.7,0.6,0.0,0.0,0.0,0.0,0.0,0.8,0.0,0.3\}$,

$/ *$ diagnosis index: $4 * /\{0.0,0.0,0.0,0.0,0.0,0.0,0.0,0.5,0.0,0.0,0.9,0.0\}$,

$/ *$ diagnosis index: $5 * /\{0.0,0.3,0.0,0.8,0.0,0.0,0.0,0.0,0.0,0.0,0.0,0.6\}$,

$/ *$ diagnosis index: $6 * /\{0.3,0.0,0.0,0.3,0.8,0.0,0.0,0.0,0.0,0.0,0.4,0.6\}$, \}

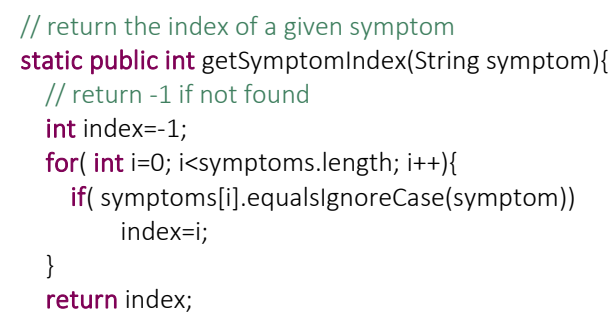

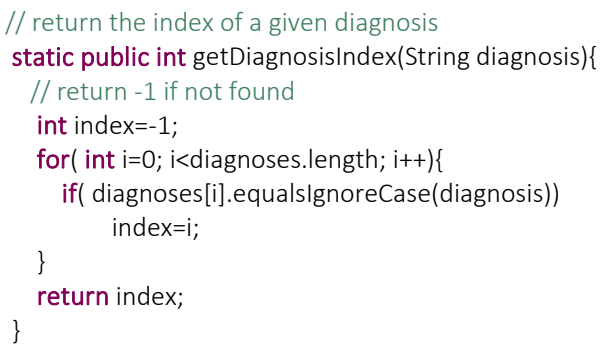

// return all diagnoses related a given symptom above a given threshold static public ArrayList getDiagnoses(String symptom, double threshold)\{ ArrayList $<$ String $>$ diagnosesList $=$ new ArrayList $<>()$;

// return all symptoms related a given diagnosis above a given threshold static public ArrayList getSymptoms(String diagnosis, double threshold)\{ ArrayList $<$ String $>$ symptomList $=$ new ArrayList $<>()$; int index= getDiagnosisIndex(diagnosis); for( int $\mathrm{i}=0$; $\mathrm{i}<$ symptoms.length; $\mathrm{i++}) \hat{\}}$ if(diag_symp[index][i]> threshold ) symptomList.add(symptoms[i]); \} return symptomList; \}

Appendix 2. Java representation of the algorithm package diagnosticalgorithm; import java.util.ArrayList; import java.util.List;

/**

* @author Irosh Fernando

* @Date 30th of June 2013

*/

public class STAlgorithm \{

static Knowledgebase KB; static PatientProfiles Patient;

static List $<$ String $>$ symptomsFound $=$ new ArrayList $<>()$; static List $<$ String $>$ symptomsToBeElicited $=$ new ArrayList $<>()$;

// To store both symptoms found and not found static List $<$ String $>$ symptomsAlreadyElicited = new ArrayList $<>()$;

// store diagnose of which symptoms are to be explored static List $<$ String $>$ diagnosesToBeElicited $=$ new ArrayList $<>($ ) ;

// Store diagnoses of which symptoms have already been explored static List $<$ String $>$ diagnosesAlreadyElicited $=$ new ArrayList $<>()$;

// set the likelihood threshold

static double likelihoodThreshold $=0.5$;

// Initialise the symptoms reported by patient at the beginning static private void initialise()\{ symptomsFound.add("Depressed Mood"); 


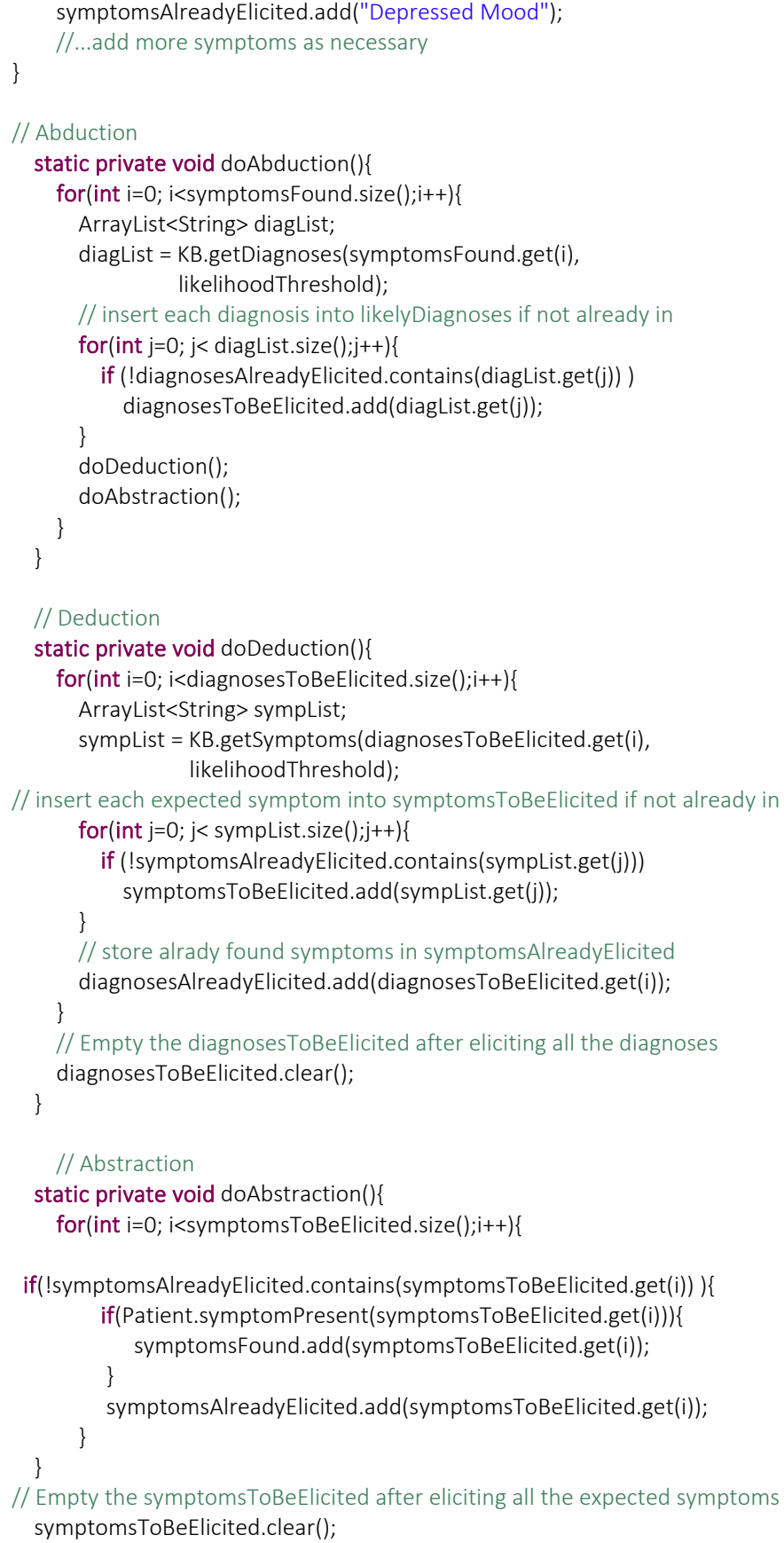

// Empty the symptomsToBeElicited after eliciting all the expected symptoms symptomsToBeElicited.clear();

\section{REFERENCES}

[1] D. A. Wolfram, "An appraisal of INTERNIST-I," Artificial Intelligence in Medicine, vol. 7, pp. 93-116, 1995.

[2] H. Mandin, A. Jones, W. Woloschuk, and P. Harasym, "Helping students learn to think like experts when solving clinical problems," Academic Medicine, vol. 72, pp. 173-179, 1997.

[3] A. S. Elstein, L. S. Shulman, and S. A. Sprafka, Medical ProblemSolving: an Analysis of Clinical Reasoning: Cambridge, MA: Harvard University Press 1978.
[4] E. Hunt, "Cognitive Science: Definition, Status, and Questions " Annual Review of psychology, vol. 40, pp. 603-629 1989.

[5] G. R. Norman, C. L. Coblentz, L. R. Brooks, and C. J. Babcook, "Expertise in visual diagnosis - a review of the literature.," Academic Medicine, vol. 66(suppl), pp. s78-s83, 1992.

[6] J. A. Reggia and Y. Peng, "Modeling diagnostic reasoning: a summary of parsimonious covering theory," Computer Methods and Programs in Biomedicine, vol. 25, pp. 125-134, 1987.

[7] P. M. Wortman, "Medical Diagnosis: An Information-Processing Approach," Computers and Biomedical Research, vol. 5, pp. 315-328, 1972.

[8] J. Stausberg and M. Person, "A process model of diagnostic reasoning in medicine," International Journal of Medical Informatics, vol. 54, pp. 9-23, 1999.

[9] E. H. Shortliffe and B. G. Buchanan, "A model of inexact reasoning in medicine," Mathematical Biosciences, vol. 23, pp. 351-379, 1975.

[10] S. Andreassen, F. V. Jensen, and K. G. Olesen, "Medical expert systems based on causal probabilistic networks," International Journal of BioMedical Computing, vol. 28, pp. 1-30, 1991.

[11] T. Chard and E. M. Rubenstein, "A model-based system to determine the relative value of different variables in a diagnostic system using Bayes theorem," International Journal of Bio-Medical Computing, vol. 24, pp. 133-142, 1989.

[12] B. S. Todd, R. Stamper, and P. Macpherson, "A probabilistic rule-based expert system," International Journal of Bio-Medical Computing, vol. 33, pp. 129-148, 1993.

[13] K. Boegl, K. P. Adlassnig, Y. Hayashi, T. E. Rothenfluh, and H. Leitich, "Knowledge acquisition in the fuzzy knowledge representation framework of a medical consultation system," Artificial Intelligence in Medicine, vol. 30, pp. 1-26, 2004.

[14] L. Godo, R. L. de Mántaras, J. Puyol-Gruart, and C. Sierra, "Renoir, Pneumon-IA and Terap-IA: three medical applications based on fuzzy logic," Artificial Intelligence in Medicine, vol. 21, pp. 153-162, 2001.

[15] T. Vetterlein and A. Ciabattoni, "On the (fuzzy) logical content of CADIAG-2," Fuzzy Sets and Systems, vol. 161, pp. 1941-1958, 2010.

[16] I. Fernando, F. Henskens, and M. Cohen, "An Approximate Reasoning Model for Medical Diagnosis," in Software Engineering, Artificial Intelligence, Networking and Parallel/Distributed Computing. vol. 492, R. Lee, Ed., ed: Springer International Publishing, 2013, pp. 11-24.

[17] M. Ramoni, M. Stefanelli, L. Magnani, and G. Barosi, "An epistemological framework for medical knowledge-based systems " IEEE Transactions on Systems, Man and Cybernetics, vol. 22, pp. $1361-1375,1992$.

[18] I. Fernando, F. Henskens, and M. Cohen, "A Collaborative and Layered Approach (CLAP) for Medical Expert System Development: A Software Process Model," in IEEE/ACIS 11th International Conference on Computer and Information Science (ICIS12), 2012, pp. 497-502.

[19] C. S. Peirce, "Illustrations of the logic of science, sixth paper-deduction, induction, hypothesis," The Popular Science Monthly, vol. 1, pp. 470482, 1878.

[20] American Psychiatric Association, Diagnostic and Statistical Manual of Mental Disorders: DSM-5: American Psychiatric Publishing Incorporated, 2013.

[21] I. Fernando, M. Cohen, and F. Henskens, "A systematic approach to clinical reasoning in psychiatry," Australasian Psychiatry, vol. 21, pp. 224-230, 2013.

[22] E. L. Kinney, "Medical Expert Systems - Who needs them ?," CHEST, vol. 91, pp. 3-4, 1987.

[23] A. K. Das, "Computers in Psychiatry: A Review of Past Programs and an Analysis of Historical Trends," Psychiatric Quarterly, vol. 73, pp. 351-365, 2002.

[24] D. Richards, "Collaborative Knowledge Engineering: Socialising Expert Systems," in 11th International Conference on Computer Supported Cooperative Work in Design, 2007. 